\title{
MEAN ESCAPE DEPTH OF SIGNAL PHOTOELECTRONS FROM AMORPHOUS AND POLYCRYSTALLINE SOLIDS
}

\author{
I.S. TILININ* , A. JABLOŃSKI AND B. LESIAK-OREOWSKA \\ Institute of Physical Chemistry, Polish Academy of Sciences \\ Kasprzaka 44/52, 01-224 Warszawa, Poland
}

Escape depth of photoelectrons leaving a target without being scattered inelastically was submitted to extensive theoretical analysis. Dependence of the mean escape depth on the $\mathrm{X}$-ray angle of incidence and the electron initial angular distribution inside the sample was considered. The Monte Cario algorithm was developed basing on a differential elastic scattering cross-section calculated within the partial-wave expansion method and a realistic electron-atom interaction potential. The mean escape depth was evaluated by means of the depth distribution function found analytically by solving a kinetic equation and by the Monte Carlo technique. The agreement between the results obtained from two methods is excellent. Elastic scattering was found to reduce considerably the escape depth. This reduction may reach up to $25 \%$ in the case of heavy elements in the practical X-ray photoelectron spectroscopy analysis. It was shown that the mean escape depth expressed in units of the electron transport mean free path is a universal function of the ratio of the inelastic to the transport mean free paths, the asymmetry parameter and the X-ray angle of incidence. A simple explicit expression for this function is proposed.

PACS numbers: 79.60.-i, 72.10. Bg, 34.80.Bm

\section{Introduction}

Signal photoelectrons escaping from a sample without a considerable energy loss have been traditionally used in surface sensitive techniques such as ultraviolet photoelectron spectroscopy (UPS) and X-ray photoelectron spectroscopy (XPS). Due to relatively small values of the inelastic mean free path (IMFP) of electrons in solids in the relevant energy range the probed depth in quantitative XPS analysis is usually of the order of a few nm. In many practical applications however it

*On leave from Moscow Engineering Physics Institute, Kashirskoye sh., 31, Moscow 115409, Russia. 
is often necessary to know the exact value of the mean escape depth (ED) of electrons from a target. Such quantity is believed to characterize most conveniently the effective thickness of the analysed top surface layer. Until recently the commonly adopted concept of the exponential decay of escape probability with depth has been used to estimate the volume of a solid target providing a major contribution to the measured intensity of a photoelectron line. This estimation is based on the assumption that elastic scattering effect can be neglected and the electron attenuation in the target is entirely due to inelastic interaction. Under this condition the particle trajectory in the medium represents a part of a straight line and the mean escape depth is equal to [1]:

$\langle z\rangle=\lambda_{\mathrm{i}} \cos \alpha$

where $\lambda_{\mathrm{i}}$ is the IMFP and $\alpha$ is the polar emission angle counted from the surface normal. Thus, any electron emitted initially towards the bulk of the target is believed not to be able to leave the sample.

Meanwhile numerous studies indicate that the ignoring of elastic scattering may cause serious errors in quantitative interpretation of the experimental data [2-7]. Elastic collisions have been found to affect noticeably the medium energy electron transport in solids and to lead to an overall decrease in the effective escape depth by several tens of percent with respect to the value predicted by Eq. (1). The theoretical studies also show that, in general, the escape probability depends strongly on geometrical conditions. This leads to a similar dependence of the mean escape depth.

In this report the mean escape depth of photoelectrons leaving the target is calculated analytically by solving a classical kinetic equation in the transport approximation [6,7] and by applying the Monte Carlo simulation method [8] based on a realistic elastic scattering cross-section [9].

\section{Theory}

The escape probability of signal photoelectrons as a function of the depth of origin is proportional to the flux density of electrons emerging from the target with a source situated at a certain depth $z_{0}$. The flux density can be found by solving a transport equation with a boundary condition irrplying that no secondaries enter the target. Suppose that a photon beam with the initial flux $F$ is incident on the flat target at the polar angle $\theta$ counted from the surface normal. Let $N(z, \Omega)$ be the flux density of photoelectrons moving at the depth $z$ in the direction $\Omega$, where $\Omega$ is the unit vector along the particle velocity so that $\mu=n_{z} \cdot \Omega=\cos \vartheta$ is the cosine of the polar angle $\vartheta$ counted from the $z$-axis with the unit vector $n_{z}$ directed inside the target. By making use of the transport approximation $[6,7]$ we have the following formulation of the secondary emission problem:

$$
\begin{aligned}
& \mu \frac{\partial N}{\partial z}=-\frac{N}{\lambda_{\mathrm{t}}}+\frac{1}{4 \pi \lambda_{\mathrm{tr}}} \int_{4 \pi} N\left(z, \Omega^{\prime}\right) \mathrm{d} \Omega^{\prime}+Q(\Omega) \delta\left(z-z_{0}\right), \\
& N(z=0, \Omega)=0 \quad \text { for any } \quad n_{z} \Omega>0
\end{aligned}
$$

where $\lambda_{\mathrm{t}}$ is the total mean free path in the transport approximation

$$
\lambda_{\mathrm{t}}=\lambda_{\mathrm{i}} \lambda_{\mathrm{tr}} /\left(\lambda_{\mathrm{i}}+\lambda_{\mathrm{tr}}\right)
$$


$\lambda_{\mathrm{tr}}$ is the transport mean free path, and the source function $Q(\mu)$ describes the initial angular distribution of the photoelectrons. For unpolarized radiation this function is given by the expression [10]:

$$
Q(\Omega)=n_{0} F \sigma_{\mathrm{ph}} f\left(\Omega, \Omega_{\gamma}\right)
$$

In the latter formula $n_{0}$ is the atomic density, $\sigma_{\mathrm{ph}}$ is the total photoelectric cross-section, the unit vector $\Omega_{\gamma}$ characterizes the direction of photon propagation, $f\left(\Omega, \Omega_{\gamma}\right)$ is the normalized differential photoelectric cross-section

$$
f\left(\Omega, \Omega_{\gamma}\right)=\frac{1}{4 \pi}\left[1-\frac{\beta}{4}\left(\cos ^{2} \Theta-1\right)\right],
$$

where $\Theta$ is the angle between the photoelectron initial momentum and the X-ray propagation direction, $\cos \Theta=\boldsymbol{\Omega} \cdot \boldsymbol{\Omega}_{\gamma}$, and $\beta$ is the asymmetry parameter.

Solution of Eq. (2) with boundary condition (3) may be expressed in terms of the Green function $G\left(\tau, \Omega \mid \tau_{0}, \Omega\right)$ :

$$
\begin{aligned}
& N(z, \Omega)=y_{0} \int_{4 \pi} \mathrm{d} \Omega_{0} G\left(\tau, \Omega \mid \tau_{0}, \Omega_{0}\right) f\left(\Omega_{0}, \Omega_{\gamma}\right) \\
& \left(y_{0}=n_{0} F \sigma_{\mathrm{ph}} \lambda_{\mathrm{tr}} \omega\right),
\end{aligned}
$$

where $\tau$ is the reduced depth

$$
\tau=z / \omega \lambda_{\mathrm{tr}}
$$

$\omega$ is the single scattering albedo [11]:

$$
\omega=\lambda_{\mathrm{i}} /\left(\lambda_{\mathrm{i}}+\lambda_{\mathrm{tr}}\right)
$$

and the Green function $G$ satisfies the transport equation and the boundary condition

$$
\begin{aligned}
& \mu \frac{\partial G}{\partial \tau}=-G+\frac{\omega}{4 \pi} \int_{4 \pi} G\left(\tau, \Omega^{\prime} \mid \tau_{0}, \Omega_{0}\right) \mathrm{d} \Omega^{\prime}+\delta\left(\Omega-\Omega_{0}\right) \delta\left(\tau-\tau_{0}\right), \\
& G\left(\tau=0, \Omega \mid \tau_{0}, \Omega_{0}\right)=0 \quad \text { for any } \quad n_{z} \Omega>0 .
\end{aligned}
$$

Should the Green function value at the surface $G\left(0, \Omega \mid \tau_{0}, \Omega_{0}\right)$ be known for negative values of $\mu<0$, one can easily calculate the flux density of signal electrons leaving the target by means of Eq. (6).

Due to linearity of the transport equation we may write the solution of Eq. (7) as the surn of two terms

$$
G\left(\tau, \Omega \mid \tau_{0}, \Omega_{0}\right)=G_{1}\left(\tau, \mu \mid \tau_{0}, \mu_{0}\right)+G_{2}\left(\tau, \Omega \mid \tau_{0}, \Omega_{0}\right)
$$

where the function $G_{1}$ does not depend on the azimuthal angle and is the solution of $\mathrm{Eq}$. (7) with the source function

$$
Q_{1}(\tau, \Omega)=(2 \pi)^{-1} \delta\left(\tau_{0}-\tau\right) \delta\left(\mu_{0}-\mu\right),
$$

while the function $G_{2}\left(\tau, \Omega \mid \tau_{0}, \Omega_{0}\right)$ satisfies the same equation except for the source function being equal to

$$
Q_{2}(\tau, \Omega)=\delta\left(\tau-\tau_{0}\right) \delta\left(\Omega-\Omega_{0}\right)-Q_{1}(\tau, \Omega)
$$

The functions $G_{1}$ and $G_{2}$ are assumed to meet the boundary condition given by Eq. (8). The explicit expression for the function $G_{1}$ can be found elsewhere (Ref. [12], p. 116). On the other hand, we have for the function $G_{2}$ : 


$$
\begin{aligned}
& G_{2}\left(\tau, \Omega \mid \tau_{0}, \Omega_{0}\right) \\
& \quad=\left\{\begin{array}{cc}
\left|\mu_{0}\right|^{-1} \exp \left(-\left|\left(\tau-\tau_{0}\right) / \mu_{0}\right|\right) & \\
\times\left[\delta\left(\Omega-\Omega_{0}\right)-(2 \pi)^{-1} \delta\left(\mu-\mu_{0}\right)\right] & \text { for }\left(\tau-\tau_{0}\right) / \mu_{0}>0, \\
0 & \text { for }\left(\tau-\tau_{0}\right) / \mu_{0}<0 .
\end{array}\right.
\end{aligned}
$$

The above resuit together with the formula for the function $G_{1}$ of Ref. [12] completely determine the Green function $G\left(\tau, \Omega \mid \tau_{0}, \Omega_{0}\right)$, and thereby the flux density $N(z, \Omega)$.

The depth distribution function (DDF) $\Phi(z, \Omega)$ describes the probability that an electron generated initially at the depth $z$ will reach the surface and leave the target in a certain direction $\Omega$ [2]. Hence the DDF is treated as the probability density with respect to both $z$ and $\Omega$ variables. This function integrated over all the escape depths and emission angles provides the total number of photoelectrons leaving the unit area of the surface per unit time. It follows from this normalization condition that the DDF is equal to the outgoing flux density of electrons multiplied by the absolute value of the cosine of emission angle $\mu$ :

$$
\Phi(z, \Omega)=-\mu y_{0} \int_{4 \pi} G\left(0, \Omega \mid \tau, \Omega_{0}\right) f\left(\Omega_{0}, \Omega_{\gamma}\right) \mathrm{d} \Omega_{0}, \quad \mu<0 .
$$

Due to lack of space we do not present the explicit expression of the DDF and confine ourselves to evaluating the mean escape depth defined by the formula

$$
\langle z\rangle=\int z \Phi(z) \mathrm{d} z\left(\int \Phi(z) \mathrm{d} z\right)^{-1}
$$

where the integration is performed from 0 to $\infty$. Substituting (11) into (12) and performing integration we find

$$
\langle z\rangle=\frac{\lambda_{\mathrm{i}} \lambda_{\mathrm{tr}}}{\lambda_{\mathrm{i}}+\lambda_{\mathrm{tr}}}(\cos \alpha+S)
$$

Here the position of the analyser is conveniently specified by the polar angle $\alpha$ and the azimuthal angle $\varphi$, counted from the plane of incidence of X-rays. The quantity $S$ is described by the ratio

$$
S=S_{1} / S_{2}
$$

where

$$
\begin{aligned}
S_{1}= & (1-\omega)^{-1 / 2}\left(\nu_{0}-1+\chi_{0}\right) \\
& +\frac{\omega \beta}{16}\left(3 \mu_{\gamma}^{2}-1\right) \int_{0}^{1} \frac{\left(x^{2}-x \cos \alpha\right)\left(3 x^{2}-1\right) H(x, \omega)}{(x+\cos \alpha)} \mathrm{d} x, \\
S_{2}= & (1-\omega)^{-1 / 2}-\frac{\beta\left(3 \cos ^{2} \Theta-1\right)}{4 H(\cos \alpha, \omega)} \\
& +\frac{\omega \beta}{16}\left(3 \mu_{\gamma}^{2}-1\right) \int_{0}^{1} \frac{x\left(3 x^{2}-1\right) H(x, \omega)}{(x+\cos \alpha)} \mathrm{d} x .
\end{aligned}
$$

In Eqs. (15) and (16) $\mu_{\gamma}$ is the cosine of the X-ray angle of incidence $\mu_{\gamma}=\cos \Theta$, $\nu_{0}$ is the positive root of the characteristic equation

$$
1=\left(\omega \nu_{0} / 2\right) \ln \left[\left(\nu_{0}+1\right) /\left(\nu_{0}-1\right)\right]
$$


and $\chi_{0}$ is the integral

$$
\chi=(1 / \pi) \int_{0}^{1} \chi(\mu) \mathrm{d} \mu,
$$

where the function $\chi(\mu)$ is defined by

$$
\chi(\mu)=\left\{\begin{array}{cc}
\operatorname{arctg} x(\mu), & x>0, \\
\pi+\operatorname{arctg} x(\mu), & x<0
\end{array}\right.
$$

and

$$
x(\mu)=(\omega \pi \mu / 2)\left\{1-(\omega \mu / 2) \ln \left[\left(\nu_{0}+1\right) /\left(\nu_{0}-1\right)\right]\right\}^{-1} .
$$

The cosine of the angle $\Theta$ is related to the emission angles $\alpha$ and $\varphi$ by the expression: $\cos \Theta=-(\cos \Theta \cos \alpha-\sin \Theta \sin \alpha \cos \varphi)$.

At this stage it is possible to compare expression (13) with Eq. (1) corresponding to the straight line approximation (i.e. when elastic scattering is neglected). One can see that accounting for elastic scattering leads to two principal effects. First of all the mean escape depth is not zero for the emission angle $\alpha=90^{\circ}$ in contrast to the value predicted by expression (1). Due to deflections in elastic collisions some of the electrons generated at depths $z \approx \lambda_{\mathrm{i}} \lambda_{\mathrm{tr}} S /\left(\lambda_{\mathrm{i}}+\lambda_{\mathrm{tr}}\right)$ can escape from the target at grazing emission angles. Secondly, the quantity $\langle z\rangle$ depends strongly on the angle $\Theta$ betweeen the X-ray propagation direction and the direction of photoemission. In particular, the largest $\langle z\rangle$ values are expected at $\Theta \approx 90^{\circ}$ and $\beta \approx 2$ and the smallest for $\Theta=0$. Analysis of expression (13) shows that the mean escape depth can be either greater or smaller than $\lambda_{\mathbf{i}} \cos \alpha$ depending on geometry considered and asymmetry of the initial angular distribution.

\section{Comparison of analytical results with Monte Carlo simulation data}

The mean escape depth was calculated by the Monte Carlo procedure for a number of elements and different geometries in the plane of incidence of X-rays. Details of the Monte Carlo algorithm are described elsewhere $[8,9]$. We note only that in the usual simulation scheme the trajectories are assumed to consist of linear steps between elastic collisions and the electron motion is approximated by the Poisson stochastic process. Elastic scattering events are modelled by a realistic differential scattering cross-section calculated from the partial wave expansion method for the Thomas-Fermi-Dirac potential. Figure 1 illustrates the correlation between the anisotropy of the initial angular distribution of electrons in the target and the mean escape depth dependence on the X-ray angle of incidence for the $4 s$ line in gold and the emission angle $\alpha=60^{\circ}$ (the asymmetry parameter $\beta=1.82$, the photoelectron energy is $491 \mathrm{eV}$ ). The ratio $\lambda_{\mathrm{i}} / \lambda_{\mathrm{tr}}$ is about 0.5 , therefore one might expect a considerable influence of the elastic scattering effect. Figure 1a displays the initial angular distribution which may be also interpreted as the angular spectrum of photoelectrons leaving the target when elastic scattering is neglected. In Fig. 1b the dependence of the mean escape depth on the $\mathrm{X}$-ray angle of incidence is shown. One can see that the minimum of the function $f(\Theta)$ corresponds to the maximum value of the escape depth. This peculiarity can be understood qualitatively if one realizes that a relatively small number of 

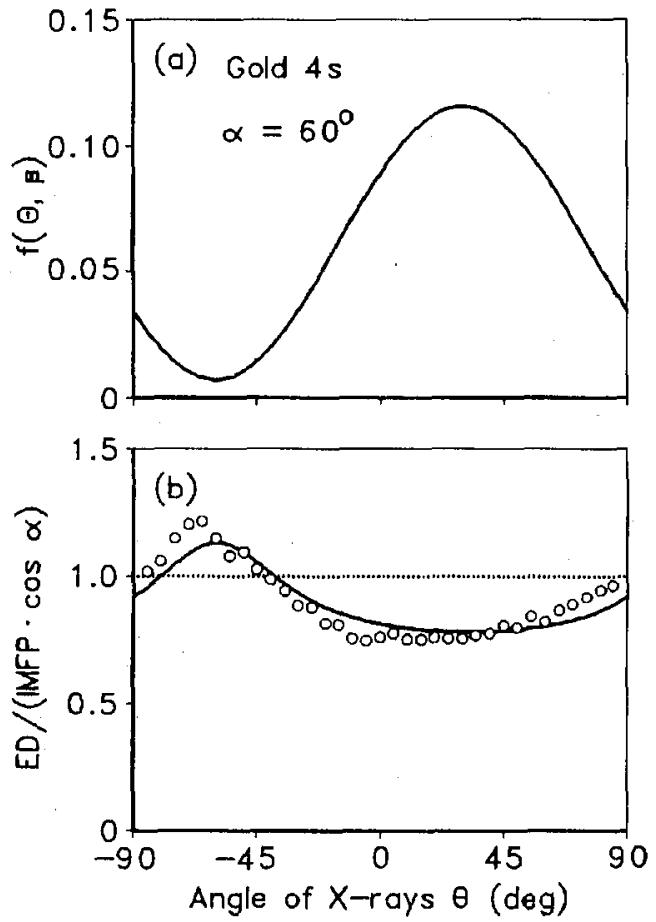

Fig. 1. (a) The initial angular distribution of photoelectrons for $4 \mathrm{~s}$ line in gold $(\beta=1.82, E=491 \mathrm{eV}) . \Theta$ is the angle between the X-ray propagation direction and the direction of photoemission $\left(\alpha=60^{\circ}\right)$. (b) The dependence of the mean escape depth (ED) (in units of the inelastic mean free path multiplied by the cosine of the emission angle) on the X-ray angle of incidence $\theta\left(\alpha=60^{\circ}\right)$, in the plane of $n$. The negative values of the angle $\theta$ correspond to the emission azimuthal angle $\Phi=180^{\circ}$.

signal electrons are moving towards the surface in the direction $\alpha=60^{\circ}$. Owing to elastic scattering some other electrons may change their momentum direction and thereby make an additional contribution to the current collected by an analyser in a narrow solid angle. Those particles, however, travel in average larger path lengths and escape from deeper layers. Therefore, they effectively increase the mean escape depth along the direction of the minimum of the initial angular distribution. In Fig. 2 the variation of the mean escape depth, expressed in units of the product of the emission angle cosine and the inelastic mean free path, with the angles $\alpha$ and $\theta$ is shown. A good agreement is observed between the analytical and Monte Carlo results in all cases except for the emission angle $\alpha=80^{\circ}$. The latter discrepancymay be attributed to a poor accuracy of Monte Carlo data at grazing emission angles when a comparatively large half cone acceptance angle $\Delta \alpha=10^{\circ}$ can affect considerably the estimated intensity.

In future extensive tabulation is planned to be made with parameters necessary for determination of the escape depth corresponding to all major photoelectron lines and most frequently used experimental geometries. 


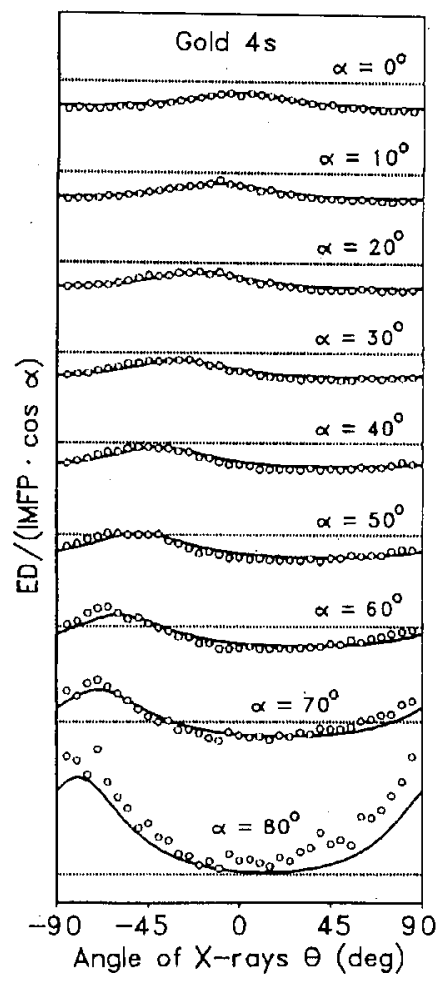

Fig. 2. The dependence of the mean escape depth (ED) on the X-ray angle of incidence $\Theta$ and the emission angle $\alpha$ in the plane of incidence. The negative values of the angle $\Theta$ correspond to the emission azimuthal angle $\Phi=180^{\circ}$. The dotted line corresponds to unity, similarly to Fig. 1.

\section{Acknowledgments}

This work has been supported by the grant 224669102 of the State Committee for Scientific Research (Republic of Poland).

\section{References}

[1] S. Hoffman, in: Practical Surface Analysis, 2nd ed., Eds. D. Briggs, M.P. Seah, Vol. 1, Wiley, Salle+Saurlander, Chichester 1990, p. 143.

[2] A. Jabłoniski, H. Ebel, Surf. Interface Anal. 11, 627 (1988).

[3] O.A. Baschenko, V.I. Nefedov, J. Electron Spectrosc. 17, 405 (1979).

[4] S. Tougaard, P. Sigmund, Phys. Rev. B 25, 4452 (1982).

[5] A. Jabłoński, Surf. Interface Anal. 14, 659 (1989).

[6] I.S. Tilinin, W.S.M. Werner, Phys. Rev. B 46, 13739 (1992).

[7] W.S.M. Wenner, I.S. Tilinin, Appl. Surf. Sci. 70/71, 29 (1993).

[8] A. Jabłoński, J. Zemek, Phys. Rev. B 48, 4799 (1993). 
[9] A. Jabłoński, Phys. Rev. B 43, 7546 (1991).

[10] R.F. Reilman, A. Msezane, S.T. Manson, J. Electron Spectrosc. Relat. Phenom. 8, 289 (1976).

[11] S. Chandrasekhar, Radiative Transfer, 1st ed., Clarendon Press, Oxford 1950, p. 6.

[12] K. Case, P. Zweifel, Linear Transport Theory, Addison-Wesley, Reading, Massachusets 1967, p. 116. 